\title{
De l'analyse des pratiques de participation citoyenne à des propositions pour une éducation à l'environnement
}

\section{Cécile Fortin-Debart et Yves Girault}

\section{OpenEdition}

Journals

Édition électronique

URL : https://journals.openedition.org/ere/2219

DOI : 10.4000/ere.2219

ISSN : 2561-2271

Éditeur

Centr'ERE

\section{Référence électronique}

Cécile Fortin-Debart et Yves Girault, « De l'analyse des pratiques de participation citoyenne à des propositions pour une éducation à l'environnement », Éducation relative à l'environnement [En ligne], Volume 8 | 2009, mis en ligne le 20 décembre 2009, consulté le 21 septembre 2021. URL : http:// journals.openedition.org/ere/2219; DOI : https://doi.org/10.4000/ere.2219

Ce document a été généré automatiquement le 21 septembre 2021.

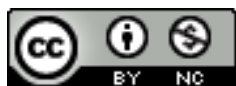

La revue Éducation relative à l'environnement est mise à disposition selon les termes de la Licence Creative Commons Attribution - Pas d'Utilisation Commerciale 4.0 International. 


\title{
De l'analyse des pratiques de participation citoyenne à des propositions pour une éducation à l'environnement
}

\author{
Cécile Fortin-Debart et Yves Girault
}

\section{Participation citoyenne et éducation au pouvoir}

1 La participation citoyenne, définie comme l'implication des individus et des groupes dans un processus de décision, est convoquée de nos jours dans de nombreux discours relatifs à l'environnement, à la gestion et l'aménagement du territoire. Mais quels en sont les usages et les pratiques? Quels détournements peut-on observer? Quels bénéfices les citoyens peuvent-ils en tirer? De nombreuses questions interpellent les différentes dimensions éthiques de la participation. À partir du constat des pratiques de participation citoyenne mobilisées aujourd'hui en France, nous proposons dans cet article de réfléchir aux modalités d'une éducation relative à l'environnement (ERE) préoccupée de participation citoyenne d'une part et d'autre part d'élaborer un certain nombre de propositions : que peut donc signifier la participation citoyenne dans le champ des questions environnementales et quelles sont les conséquences en terme d'ERE, d'un point de vue théorique et pratique, dans le cadre de l'enseignement scolaire? Nous nous positionnons ici dans le champ de recherche et de pratique de l'éducation relative à l'environnement, dans une perspective plus large que celle des instructions officielles françaises recommandant la généralisation d'une éducation au développement durable dans l'enseignement scolaire, principalement en raison du flou et des dérives liés à la notion de développement durable.

Dans le cadre de l'enseignement scolaire, éducation (ou instruction selon les auteurs) et citoyenneté sont intimement liées. Depuis Condorcet, «l'école obligatoire et publique française continue de poser comme objectif premier de l'école publique la formation du 
citoyen » (Crémieux, 1998, p. 91). Les savoirs, les savoir-faire, la culture transmise par et à l'école ont pour première finalité la formation de citoyens capables de débattre des objets qui les concernent et de choisir leurs représentants dans un espace public où ils sont égaux (Audigier, 1999). L'éducation à la citoyenneté est un champ largement étudié et nous ne prétendons pas apporter ici une nouvelle étude englobant tous les aspects de ce champ. Pour cela, nous renvoyons à deux auteurs qui ont nourri la réflexion présentée ici, et plus particulièrement l'ouvrage de Crémieux (1998) et les différents travaux d'Audigier $(1999,2006)$. Nous souhaitons cependant attirer l'attention du lecteur sur le fait qu'au-delà des discours et des mots, de nombreux auteurs convergent pour faire un constat plutôt pessimiste : " la formation du citoyen, bien qu'associée à l'école obligatoire depuis plus d'un siècle et présentée comme son principal objectif, reste plus formelle que réelle » (Crémieux, 1998, p. 112). Par ailleurs, l'éducation à la citoyenneté peut prendre plusieurs formes, de la « reprise en main des jeunes pour lutter contre les comportements déviants " (Crémieux, 1998, p. 14) à l'éducation au droit centrée sur des aspects juridiques et sur les droits de l'Homme, à travers des thématiques telles que le racisme, la discrimination ou autres.

Ce n'est pas dans ces perspectives que nous inscrivons notre réflexion, mais davantage dans celle d'une éducation au pouvoir (Audigier, 2006), qui vise à donner des compétences pour participer à la vie de la cité (vie publique, vie politique, vie quotidienne). Avant d'analyser quelles formes peut prendre une ERE qui s'inscrit dans cette éducation au pouvoir, nous allons justement nous interroger sur la nature de ce pouvoir qu'ont les citoyens face aux questions environnementales. Quelles formes de participation citoyenne sont organisées à l'heure actuelle en France? La nécessaire clarification préliminaire de ce contexte de la démarche participative nous amènera par la suite à nous questionner sur le champ des possibles pour une ERE qui se donne comme objectif principal de permettre une réelle participation citoyenne à l'encontre de l'approche comportementaliste très dominante en France.

\section{Constats sur la participation citoyenne en France}

De nos jours, la participation citoyenne est invoquée à travers de nombreux dispositifs hétérogènes émergeant qui cherchent à associer les citoyens « ordinaires » - ni experts de la technique, ni professionnels de la politique - à la discussion des enjeux collectifs et à l'élaboration des politiques publiques (Nez, 2006). De nombreux textes internationaux relatifs aux questions environnementales mettent en avant cette notion de citoyen acteur, par exemple dans le chapitre 23 de l'Agenda 21 rédigé à l'occasion du Sommet de la Terre de Rio (1992) : «l'un des principaux éléments indispensables à la réalisation du développement durable est une large participation du public à la prise de décisions ». Que tout un chacun puisse participer aux décisions élaborées en matière d'environnement et de développement durable ne fait donc quasiment aucun doute chez la plupart des acteurs concernés. On peut en effet observer un lien fort entre la mise en œuvre du développement durable et les processus de participation des citoyens dans la gestion territoriale, à différentes échelles et dans des domaines variés (Joliveau et Amzert, 2001). La question de la participation citoyenne se retrouve ainsi sur le terrain dans un foisonnement d'innovations et de procédures avec les arguments suivants: les solutions sont souvent pérennes et efficaces, en tout cas socialement acceptables et le dialogue entre les acteurs est favorisé. 
5 La participation citoyenne s'invite donc dans de nombreux discours de manière insistante sinon obsédante (Schnapper et Bachelier, 2000), dans une diversité de termes employés: développement participatif, démocratie participative, démocratie de proximité, démocratie locale, développement communautaire, etc.

6 Au-delà du flou, certains auteurs critiquent également le décalage entre les ambitions affichées et les objectifs atteints. Malgré les objectifs annoncés, il semblerait que la plupart de ces démarches s'apparentent bien plus à un processus d'information du public qu'à des moments de démocratie ou de réelle co-construction collective des projets politiques (Colin, 2007). Dans certains cas, on peut même considérer qu'il s'agit d'une photographie de l'état de l'opinion, tels des supers-sondages améliorés, qui ne permettent pas une réelle confrontation de porteurs d'avis (Colin, 2007), ce qui conduit ce dernier auteur à dénoncer par ailleurs le manque d'objectifs clairs et de moyens qu'elle considère comme pauvres sinon inexistants (Colin, 2007).

7 En allant plus loin dans la critique, si on regarde de plus près différentes initiatives récentes mises en œuvre pour faire participer les citoyens dans des décisions relatives à l'environnement ou au développement durable, on observe deux tendances : au pire, les dispositifs mis en place relèvent de la "manipulation »; au mieux, ils s'inscrivent dans une " coopération symbolique " pour reprendre les expressions proposées par Arnstein (1969). Si l'échelle mise en place par cette sociologue nous permet de bien caractériser ce qui se fait actuellement en France, la pertinence constante et très partagée de l'utilisation de cette échelle (de Carlo, 1996; Donzelot et Epstein, 2006 ; Rayssac et La Gueronniere, 2006, entre autres) permet de souligner que depuis 40 ans, malgré les discours et les nombreuses initiatives, on n'observe pas vraiment d'évolution dans les pratiques. Précisons que cette échelle a été élaborée suite à la mise en place de dispositifs de participation par les municipalités de certaines villes du nord des ÉtatsUnis dans le cadre de programme de rénovation urbaine de quartiers « défavorisés ». Le caractère factice des dispositifs rajouta aux malaises de ces populations locales et se traduisit en émeutes sanglantes sur la période de 1963 à 1968. Cette échelle qui selon Sherry Arnstein traduit une volonté de clarification «distingue trois niveaux correspondants à différents registres de pratiques» (Donzelot et Epstein, 2006, p. 7) : un premier niveau de non participation, un deuxième niveau de coopération symbolique et enfin un dernier niveau qui correspond au pouvoir effectif des citoyens. Nous allons à présent préciser et illustrer ces trois niveaux.

\section{La non-participation}

Arnstein dénonçait déjà en 1969 un premier niveau de participation qu'elle qualifiait de non-participation et qui relevait de la manipulation. Pour Donzelot et Epstein (2006, p.7), cette forme existe toujours et se définit de la manière suivante: "ce qui est qualifié de participation vise dès lors exclusivement à obtenir le soutien du public, au travers de techniques relevant de la sphère de la publicité et des relations publiques ». C'est donc une approche informative qui cherche à faire la promotion de certaines décisions dictées par les experts, les élus et l'administration, de manière à déclencher un processus d'adhésion ou encore à « vendre » des comportements favorables que tout un chacun doit adopter. Ce processus peut être illustré avec l'exemple actuel du tri des déchets tel qu'il a été mis en place en France (Jolivet, 2001). En effet, le traitement et l'élimination des ordures ménagères nécessitent la participation des «ménages » pour 
utiliser au mieux les infrastructures de collecte sélective, ce qui relève d'une implication librement consentie vis-à-vis d'une politique venue d'en haut (Jolivet, 2001). De nombreux exemples médiatiques s'inscrivent également dans cette perspective. Des campagnes orchestrées par des personnages médiatiques, qui apportent une caution scientifique ou pseudo-scientifique, interpellent le citoyen pour qu'il "participe" à l'effort collectif: chacun en achetant des ampoules basse consommation contribue à lutter contre le réchauffement climatique. De manière caricaturale, c'est ce discours qui est aujourd'hui véhiculé dans les médias et qui en appelle le plus souvent à la responsabilité individuelle des citoyens, dans leur vie quotidienne, leurs actes d'achat, etc.

\section{La coopération symbolique}

9 Arnstein distinguait en 1969 une autre forme de participation, qu'elle définissait comme une coopération symbolique. Elle qualifiait ce niveau de participation de simple rituel sans conséquence puisqu'il n'y avait aucun engagement réel quant à la prise en compte des attentes, suggestions, propositions exprimées par les personnes consultées. De nos jours, nous rencontrons très souvent cette forme de participation qui « consiste à autoriser ou même inviter des citoyens à donner des conseils et à faire des propositions, mais en laissant ceux qui ont le pouvoir seuls juges de la faisabilité ou de la légitimité des conseils en question » (Donzelot et Epstein, 2006, p. 7).

10 Cette participation consultative existe par exemple avec toutes les formes de représentation de la société civile dans les instances de propositions que sont les différents conseils mis en place par les gouvernements et les commissions diverses et variées dont le nombre ne cesse de croître et qui apparaissent souvent dès qu'un problème est médiatiquement mis au-devant de la scène (par exemple, commission mise en place suite au scandale médiatique et judiciaire de l'affaire Outreau). Blondiaux et Sintomer (2002) citent à titre d'illustration le comité national d'éthique (1983), la commission de la nationalité (1987), le Conseil national du sida (1989), le haut conseil de l'intégration (1990), la commission nationale du débat public (1995), etc. À un niveau plus local, ce sont également les commissions extramunicipales qui invitent les habitants d'une ville à exprimer leurs attentes et leurs propositions.

11 Ces derniers auteurs estiment que "c'est dans le domaine de l'environnement et de l'aménagement du territoire que les choses sont allées le plus vite et le plus loin " (Blondiaux et Sintomer, 2002, p. 19). Plusieurs exemples peuvent illustrer cette forme de participation consultative: la commission française du développement durable (1992), le Conseil national du développement durable (2003) et enfin plus près de nous, le Grenelle de l'environnement mis en place en 2007.

12 On retrouve également la participation consultative dans les nombreux dispositifs que sont les conférences de consensus, les ateliers de scénarios, les noyaux d'intervention participative, les jurys de citoyen, etc. Ces dispositifs permettent à des citoyens de débattre entre eux et de convoquer des experts pour les éclairer dans le but de produire un rapport qui présente des recommandations, des solutions aux problèmes soulevés, des orientations souhaitables, etc.

13 Cette forme de participation reste cependant une "démocratie participative faible ", car détachée de la prise de décision et encastrée dans une logique de délégation du pouvoir pour reprendre les propos de Nez (2006). Elle consacre en effet le rôle des élus 
qui restent au centre de la décision (Nez, 2006) et renvoie les citoyens aux rôles d'observateurs, de témoins et non de réels acteurs (Colin, 2007). Sintomer (2006, p. 30) fait le même constat, dénonçant une participation spectacle et une démocratie d'opinion où les responsables politiques « font librement la synthèse de la discussion, pratiquant une «écoute sélective » des arguments qu'ils intégreront ou non ». En fait, la question centrale de la participation consultative est le devenir de l'expression citoyenne : est-elle réellement prise en compte dans les processus de décision? Pour la plupart des auteurs cités, l'influence des citoyens sur la décision est rare. Dans les cas les plus ambigus, l'expression citoyenne n'a pas alimenté le processus politique, mais aux yeux du public elle le légitime et donne une image positive de la décision publique. Le risque est « la création de structures assez coupées des citoyens, où il est davantage question d'image que [...] d'association à la décision » (Sintomer, 2003, p. 139).

\section{Le pouvoir effectif des citoyens}

Si en France, c'est bien la " coopération symbolique » qui est largement représentée, il faut cependant nuancer ce propos avec les quelques expériences d'aménagement urbain et de budget participatif mises en œuvre dans quelques villes françaises. Avec ce type de dispositif, on atteint le troisième niveau de participation distingué par Arnstein (1969) qui constitue un réel pouvoir effectif des citoyens. Ici, l'espace de participation et de discussion est connecté à l'espace de décision et comme le souligne Nez (2006), les citoyens ont le pouvoir de discuter des enjeux collectifs et de participer à l'élaboration des politiques publiques. Cette dimension participative a des antécédents, en particulier dans les thématiques autogestionnaires des années 1960 et 1970 (Sintomer, 2006) revendiquées par tout un pan de la gauche politique, syndicale et associative. Puis, ces théories ne semblaient plus au goût du jour et c'est une nouvelle problématique qui apparaît dans les années 1980 et 1990 sous l'influence du budget participatif mis en place à Porto Alegre au Brésil. Ce dispositif complexe permet aux citoyens qui le souhaitent de participer à la définition du budget communal, selon des règles co-élaborées par l'exécutif municipal et les participants (Sintomer, 2006). Ces dispositifs ont ensuite été testés et mis en place dans plusieurs villes européennes: Séville, Rome, Berlin, etc. (Nez, 2006).

En France, c'est dans le cadre de la politique de la ville que s'expriment ces cas de réelle participation citoyenne: "la politique de la ville n'a jamais cessé d'encourager en théorie l'implication des habitants des quartiers sans que cette injonction participative soit toujours suivie des résultats escomptés » (Blondiaux et Sintomer, 2002, p. 21). Nez (2006) cite une dizaine d'expériences de ce type en France, où des conseils de quartier " co-décident » avec les élus et les services techniques du budget et des investissements qui concernent leur quartier : à Morsang-sur-Orge dans l'Essonne, dans les 14e et 20e arrondissements de Paris, etc. Ce type de dispositif repose sur l'idée que " pour mieux gérer, il faut gérer avec le citoyen, dans la proximité [...] Les habitants ont des savoirs pratiques : où doit passer la ligne de bus, ce qui convient le mieux pour les jeux d'enfants, etc.» (Sintomer, 2003, p.137). Paoletti (2003, p. 153) remarque que la proximité avec «un nombre d'habitants défini sur un territoire restreint" rend " virtuellement possible l'approche de l'idéal démocratique du pouvoir au peuple ».

Là aussi, nous devons porter un regard critique sur ces procédures comme nous le rappelle Paoletti (2003, p. 159) qui remarque que dans certains cas, "les instances 
participatives de quartier s'apparentent à une simple déconcentration de l'administration municipale, les conseils de quartier servant d'interlocuteurs de base aux habitants, parfois de simples guichets ». Nez (2006) estime quant à elle que la limite du processus est de n'inclure dans le budget participatif que les petites infrastructures de quartier, mais pas les grands projets et équipements urbains. Au final, malgré quelques expériences abouties de réelle participation citoyenne, Jouve (2006) estime que la politique de la ville en France se situe, dans le meilleur des cas, au niveau de la coopération symbolique.

17 Nous retiendrons donc que la participation citoyenne en France dans le cadre des questions d'environnement semble exister sous deux formes :

- une coopération symbolique qui prend la forme d'une participation consultative sur des sujets larges et variés;

- une participation dans des processus locaux d'aménagement du territoire, qui va du contrôle à la codécision, mais qui reste isolée à quelques cas et ne caractérise en aucune manière la gestion des affaires publiques locales en France.

Ces différentes formes de participation constituent néanmoins autant de pistes pour compléter ou offrir des alternatives aux procédures traditionnelles de la démocratie représentative (Blondiaux et Sintomer, 2002). Mais ce constat des pratiques sur le terrain reste en décalage avec les discours d'intention. Pourtant, depuis une trentaine d'années, et comme nous l'avons déjà dit, la participation citoyenne est fortement valorisée dans les discours politiques, et on assiste parallèlement à une réelle volonté citoyenne de participer à la décision publique. Citons par exemple l'émergence de différents groupes de pression (Greenpeace, Attac, les altermondialistes, etc.) qui s'invitent à la table des négociations, avec les limites que l'on peut avancer par exemple «cette logique du rapport de force» que dénonce Mothé (2006, p. 42). D’autres initiatives sont peut-être moins politiques et donc moins polémiques, mais permettent de mettre en avant une expression citoyenne qui souhaite de plus en plus intervenir dans les processus de décision, notamment en ce qui concerne les questions scientifiques controversées. Citons à titre d'illustration la Fondation Sciences Citoyennes, qui est une association créée en 2002, et dont l'objectif est de favoriser et de prolonger le mouvement actuel de réappropriation citoyenne et démocratique de la science, afin de la mettre au service du bien commun.

19 Les initiatives semblent donc nombreuses et variées. Alors pourquoi la situation de la participation citoyenne ne semble pas évoluer, malgré les discours d'intention? $\mathrm{Ce}$ n'est pas le sujet ici de répondre à cette question, nous retiendrons néanmoins les pistes de réflexion avancées par certains auteurs comme cette culture française de la politique, marquée par une délégation du pouvoir aux élus et ancrée dans les principes d'un gouvernement représentatif (Manin, 1996).

20 À l'instar de Donzelot et Epstein (2006), on peut donc reconnaître qu'en France, la participation est davantage un exercice incantatoire ou une exigence théorique qu'une pratique effective et que c'est la consultation dans un principe de coopération symbolique qui est largement dominante dans les pratiques. Dans le cadre d'une démarche participative, nous retiendrons que les citoyens ont donc en France la possibilité de « maximiser leur droit de vote en s'invitant dans les débats publics et en y intervenant quand on les sollicite» (Mothé, 2006, p. 38).

21 Il faut donc d'une part reconnaître les limites de telles approches consultatives comme nous l'avons fait précédemment, mais en même temps souligner leurs apports. Elles 
peuvent dans certains cas alimenter les prises de décision et la plupart du temps, elles participent à la construction d'une "intelligence collective» améliorant les compétences des personnes participantes ou observatrices (Callon et al., 2007). Surtout, elles mettent en avant la capacité des citoyens à prendre part à la décision publique et à délibérer de problèmes complexes, loin de l'hypothèse d'un public irrationnel (Callon et al., 2007). Dans cette perspective, les savoirs construits par les citoyens peuvent être tout aussi légitimes que ceux des experts pour éclairer la prise de décision. Désautels (1998, p. 20) illustre cela de manière très claire : « dans de nombreux cas, des citoyens et des citoyennes ordinaires ont contribué de manière significative à la définition et à la résolution de controverses sociotechniques ». Désaultels (1998) avance des exemples, notamment celui des activistes atteints du SIDA qui sont devenus des acteurs et des actrices crédibles autant dans l'orientation des recherches sur cette maladie que dans son traitement. Il cite un certain nombre d'auteurs anglo-saxons qui ont travaillé sur ce sujet-là (Wynne, 1996 ; Blume, 1997) et également des auteurs français (Barthe et Rémy, 1997) qui ont montré «qu'on peut difficilement raisonner sur un mode binaire, un public d'un côté, des savants de l'autre » (Barthe et Rémy, 1997, p. 76).

Hart (1992) de son côté recense de nombreux projets dans différents pays où des enfants intègrent de réels processus de participation et souligne les possibilités et les bénéfices de telles démarches à la fois pour le développement des enfants, mais aussi pour la gestion de la cité. En s'inspirant largement de l'échelle d'Arnstein (1969), il montre que pour les enfants aussi il existe une graduation de différents niveaux de participation possible, de la manipulation à la réelle participation. Reproduite ici de manière schématique, Hart (1992) propose cette graduation : les enfants sont informés ; les enfants sont consultés; les enfants co-décident avec des adultes qui ont initié le projet et enfin, les enfants initient le projet et co-décident avec des adultes.

Ce tableau des différentes formes de participation citoyenne et qui au demeurant ne prétend nullement à l'exhaustivité, nous invite à proposer une typologie d'approches en ERE susceptibles d'induire de telles démarches participatives. La graduation proposée par Hart (1992) nous semble pertinente, car elle aborde le sujet de la participation et de ces différentes formes avec les enfants, mais elle reste centrée sur le seul processus participatif. Nous souhaitons pour notre part, à partir du constat effectué ci-dessus, engager la réflexion sur les processus éducatifs qui seraient susceptibles de favoriser différentes formes de participation. Dans la typologie proposée, nous développerons plus spécialement deux visées qui nous semblent répondre plus particulièrement aux défis contemporains de la participation citoyenne.

\section{Éducation relative à l'environnement et participation citoyenne : le champ des possibles}

Nous proposons ici de caractériser trois formes d'ERE avec une typologie qui permet de mettre en perspective les différentes modalités de participation que nous avons pu constater précédemment autour de trois notions clés : l'adhésion, la délibération et l'émancipation. 


\section{L'éducation relative à l'environnement behavioriste et positiviste visant une adhésion des apprenants}

Le premier niveau caractérisé par une approche très comportementaliste et l'absence de réelle participation est associé aux courants d'ERE behavioriste et positiviste qui visent des changements de comportements à partir des informations et des recommandations formulées par les experts. Il s'agit du deficit model (Irwin, 2001) qui consiste à apporter des connaissances aux citoyens, connaissances élaborées par des experts (chercheurs, techniciens, spécialistes, etc.) qui ont la maîtrise nécessaire pour éclairer à la fois les citoyens et les politiques. À titre d'illustration, nous avions étudié l'exemple d'une association d'ERE implantée dans le département des Ardennes qui développe des activités pédagogiques avec le syndicat intercommunal de collecte et de tri des ordures ménagères en direction des écoles avec comme objectif de sensibiliser les habitants aux méthodes de collecte du syndicat. Cette forme d'ERE est peut-être la plus répandue dans les médias, mais aussi dans le cadre de l'enseignement scolaire selon un constat que nous avions récemment effectué (Fortin-Debart, 2006). Les deux autres formes que nous proposons nous semblent cependant bien plus pertinentes pour répondre aux défis d'une véritable participation.

\section{L'éducation relative à l'environnement à visée délibérative}

En rapport avec le deuxième niveau de l'échelle d'Arnstein (1969), on peut envisager une ERE qui s'intègre dans les processus de consultation et cherche à donner les meilleurs outils possibles aux citoyens pour participer à cette consultation. Nous proposons de définir cette forme d'ERE dont l'objectif est la formation d'une opinion publique éclairée, susceptible de participer aux processus consultatifs, comme une ERE à visée délibérative. Il s'agit donc de développer les compétences nécessaires pour «maîtriser » une question environnementale et pour fournir des avis ou des critiques éclairés qui pourraient venir alimenter ou influencer la décision finale des élus et, ou de l'administration. Deux dimensions doivent être envisagées de manière complémentaire : une dimension éducative qui s'inscrit ensuite dans une dimension politique où l'expression citoyenne s'invite ou est invitée à alimenter le processus de décision. Dans ce sens, on peut parler d'une "expertise citoyenne" en faisant un parallèle avec l'expertise scientifique qui est censée alimenter la prise de décision (Roqueplo, 1997). L'ERE à visée délibérative repose sur une co-construction des savoirs par les citoyens, où l'expert est éventuellement convoqué pour éclairer un point ou apporter une information que les citoyens jugent pertinente d'avoir.

Ce positionnement rejoint les principes d'une démocratie délibérative qui s'appuie sur un ensemble de propositions théoriques qui se sont multipliées à partir de la fin des années 1980 et qui prennent leurs sources dans les travaux d'Habermas (1992) et de Rawls $(1995,2003)$. Dans la démocratie délibérative, "le moment décisif n'est pas l'élection, mais la formation de l'opinion publique » (Blondiaux et Sintomer, 2002, p. 23). Il s'agit d'institutionnaliser « de la délibération sur le modèle trivial, tel qu'on le trouve à l'état de nature dans les interstices de la vie ordinaire pour que la connaissance des individus, leur expérience, leur jugement s'imbriquent dans les décisions de la société » (Mothé, 2006, p. 38). C'est tout le principe de l'espace public défini par Habermas $(1962,1992)$ qui soumet à la raison critique des citoyens les 
processus de décision, à l'instar des cafés ou salons des XVIIe et XVIIIe siècles qui soumettaient le pouvoir aristocratique à la critique de la bourgeoisie naissante. L'ERE envisagée dans cette perspective contribue alors à la formation d'une intelligence citoyenne (Hansotte, 2005) comme contre pouvoir, une citoyenneté de résistance qui peut dénoncer, négocier, résister...

D'un point de vue éducatif, il s'agit non plus de transmettre un savoir, mais d'apprendre aux citoyens la manière dont se construisent les savoirs (contextes, obstacles, influences, etc.) afin qu'ils puissent s'approprier les différents éléments du débat et comprendre les controverses émergentes. La démarche épistémologique y est privilégiée, car elle rapproche la connaissance de ses racines idéologiques ou métaphysiques, de son histoire, de ses controverses, du champ de force dans lequel elle est engendrée, vérifiée, affirmée ou combattue, des valeurs et intérêts qu'elle affaiblit ou renforce dans la société (Perrenoud, 1998). L'ERE à visée délibérative rejoint ainsi une certaine dimension de l'éducation science - technique - société (STS) puisqu'elle implique notamment « le développement de valeurs et d'habilités relatives à la prise de décision quant à l'utilisation de la science et de la technologie en regard d'une certaine qualité de société » (Sauvé, 1997, p. 66). De nombreux travaux alimentent ce champ de recherche et de pratique de l'éducation STS, en particulier dans les pays anglo-saxons (Driver et al., 2000; Kolsto, 2001). En France, quelques chercheurs s'intéressent également à ce lien entre une ERE et un enseignement scientifique "socialisé" notamment à travers l'enseignement des questions socialement vives (Legardez et Simonneaux, 2006).

\section{L'éducation relative à l'environnement à visée « émancipatrice »}

29 On peut enfin envisager une troisième voie, une ERE qui ne dépend plus des processus classiques de consultation, mais cherche à intégrer la réelle participation citoyenne (niveau 3 dans l'échelle d'Arnstein, 1969). Dans cette orientation, c'est la mobilisation par, dans et pour l'action qui est favorisée, et l'objectif est la transformation des réalités socio-environnementales qui posent problème. Nous proposons de définir cette forme d'ERE dans le cadre d'une perspective de participation citoyenne comme une ERE à visée émancipatrice. On retrouve alors ici d'autres présupposés pédagogiques pour une ERE, celle d'un courant éducatif qui vise la transformation sociale. L'objectif n'est pas de participer ou d'influencer les processus de prise de décision en mettant en avant une expression ou expertise citoyenne comme nous venons de le voir, il s'agit ici d'une mobilisation pour l'action.

L'ERE à visée émancipatrice s'inscrit dans une approche socioconstructiviste où la coconstruction de savoirs va guider l'action. Cette approche s'inscrit dans le courant de la critique sociale tel qu'il a été défini par Robottom et Hart (1993) et qui vise le développement d'un engagement dans l'action, individuellement et collectivement, afin d'améliorer l'environnement physique et social. L'approche de la critique sociale repose principalement sur des processus d'investigation des réalités environnementales, sociales et éducationnelles qui posent problème dans le but de transformer ces dernières. Plus précisément, les auteurs précisent que les expériences montrent que ces investigations et cet engagement dans l'action sont plus efficaces quand ils sont collectifs plutôt qu'individuels et en relation avec l'environnement proche (Robottom et Hart, 1993). Les investigations s'inscrivent par ailleurs dans une 
perspective critique, c'est-à-dire que l'apprenant doit pouvoir développer une analyse critique des valeurs et des intérêts sous-jacents, rendant ainsi visible la nature conflictuelle des réalités environnementales (Robottom et Hart, 1993).

31 En résumé, le courant de la critique sociale repose d'une part sur des investigations menées collectivement à propos de réalités environnementales, sociales et éducationnelles qui posent problème dans un environnement proche et d'autre part sur engagement dans le changement de ces réalités. Les investigations impliquent un processus d'analyse critique des valeurs et des intérêts sous-jacents, dont la présence caractérise la nature conflictuelle des questions environnementales. Le courant de la critique sociale inscrit donc le rapport à l'environnement dans le domaine de la critique et du changement social. Dans ce courant où l'éducation sociale est particulièrement mise en avant, "l'ERE tend à favoriser la coopération dans l'apprentissage et dans l'exercice de l'action environnementale » (Sauvé, 1997, p. 105).

Plus généralement, on aborde ici certaines théories sociales de l'éducation dans lesquelles, «l'éducation doit jouer un rôle important dans la reconstruction de la société » (Bertrand, 1998, p.166) et notamment la pédagogie de la libération développée par Freire (1972). L'ensemble de ces principes rejoint également l'idée d' empowerment, terme anglais qui renvoie à la notion d'autonomie, et à la capacité des personnes ou groupes de personnes de prendre en charge eux-mêmes leur destinée économique, professionnelle, familiale et sociale. L'empowerment constitue le processus par lequel un individu ou un groupe va acquérir les moyens et les capacités de renforcer sa capacité d'action, de s'émanciper (Bacqué et Carrel, 2005). Cette notion désigne donc un renversement des rapports de domination entre l'État et la société civile, notamment les groupes sociaux les plus défavorisés (Jouve, 2006).

Certains auteurs ont abordé des stratégies d'ERE à la lumière du concept d' empowerment, et notamment Carine Villemagne (2005) qui explore le champ de l'ERE en milieu communautaire à travers son travail de recherche sur le programme d'action environnementale Éco-quartier dans la ville de Montréal. Elle en conclue entre autres que les communautés présentent un contexte favorable au développement d'un processus d'empowerment de leurs membres intégrant des préoccupations sociales, culturelles, communautaires et écologiques, mais aussi intégrant des valeurs de solidarité, de coopération, de participation et de responsabilité, etc. (Villemagne, 2005).

\section{Pour conclure : quelles perspectives dans l'enseignement scolaire?}

Au cours de différents projets menés en contexte scolaire, nous avons tenté de mettre en œuvre ces deux dernières visées, soit l'émancipatrice ou la délibérative. Nous avons par exemple expérimenté une forme d'ERE délibérative dans le cadre d'une rechercheaction que nous avons menée en partenariat avec une association de culture scientifique, la Fondation 93, dans le cadre du dispositif PICRI proposé par la Région Îlede-France (Partenariat Institution Citoyens pour la Recherche et l'Innovation). L'objectif était de permettre à des élèves de collèges et de lycées implantés en SeineSaint-Denis de co-construire une expertise sur une question qu'ils avaient eux-mêmes définie dans le cadre du thème de la mobilité dans leur département. Les élèves, à partir d'une première réflexion sur leurs propres pratiques et représentations sociales, ont mené des investigations auprès d'acteurs locaux, d'experts, d'habitants et ont été invités à exprimer des constats, des avis, voire des attentes et des propositions sur la 
base d'une réflexion argumentée construite sur cette confrontation entre des savoirs d'experts, des savoirs expérientiels, des pratiques sociales, etc. Les premiers résultats de cette recherche-action soulignent la capacité de ces jeunes à s'engager dans une question qui les préoccupe fortement et également la manière dont ce travail leur a fait changer de regard sur leur territoire et sur leur place dans ce territoire (Girault et al., 2008).

Ce projet de recherche-action-formation qui n'entre dans aucun programme institutionnalisé et/ou généralisé d'ERE est-il pour autant très innovant? En effet, on peut trouver dans la bibliographie depuis une trentaine d'années des projets similaires. On peut citer par exemple le modèle de recherche-action pour la résolution de problèmes communautaires proposé par Stapp et coll. dès 1988 et qui consiste à engager "les élèves dans un projet collectif de résolution d'un problème social ou environnemental qu'ils ont eux-mêmes repéré dans leur milieu de vie communautaire, et qu'ils mènent en cogestion avec l'enseignant, en alliant l'action à la réflexion critique » (Sauvé, 1997, p. 234). On peut également citer les travaux de Hart qui en 1992 décrivait plusieurs projets menés dans différents pays notamment au Royaume-Uni où, dans les années 1970 et 1980, se sont multipliés des exemples de projets communautaires menés à l'école primaire. En participant à des petits projets réalistes, les élèves et leurs enseignants intégraient les processus de gestion de la communauté et d'aménagement du territoire à un niveau très local.

Avec cet exemple, Hart (1992) met en avant les leviers favorables à de tels projets : le degré élevé de liberté et d'autonomie des chefs d'établissements des écoles primaires britanniques, la volonté politique d'intégrer les populations locales aux questions d'aménagement, l'engagement d'un personnage clé ayant des responsabilités politiques dans le domaine de l'éducation et ayant favorisé de tels dispositifs et surtout l'aide apportée par des acteurs extérieurs comme les Urban Studies Centres (Centres d'études urbaines), où les élèves et les habitants viennent trouver une aide méthodologique et un accompagnement dans leurs investigations quand ils en ont besoin. En compilant les études menées par les élèves et les habitants, ces centres sont également devenus, selon cet auteur, des lieux de référence et de savoirs co-construits exploités par la communauté dans son ensemble.

Ces projets relèvent donc de l'innovation, car ils ne peuvent pas être programmés et institutionnalisés tant leur développement relève d'une combinaison de facteurs favorables (personnes motivées, partenaires extérieurs à l'école pouvant favoriser et soutenir la démarche). Ces projets nécessitent également un réel ancrage local, une réponse à un contexte très localisé et ne peuvent ainsi se voir dotés d'une méthodologie générale et applicable par tous. Notre recherche-action menée dans le cadre des PICRI de la Région Île-de-France a confirmé l'ancrage local indispensable, ancrage déjà souligné dans le contexte de l'éducation à la citoyenneté : «les projets les plus porteurs sont ceux qui se centrent sur la personne de l'élève, sur son cadre de vie; c'est dans le concret de ces actions que le jeune peut découvrir ce que recouvre la notion de citoyenneté sociale» (Baillon, 1993, p. 163). Il est intéressant de souligner que cette dimension locale est un principe fondateur d'une éducation à la citoyenneté dans certains pays qui favorisent "l'autonomie des établissements et, ou des autorités locales pour adapter les curriculums et autres programmes établis par les autorités centrales, aux contextes locaux » (Audigier, 2006, p. 42). 
Cette même recherche-action décrite précédemment nous a également permis de mettre en avant la nécessité d'accompagner les enseignants dont les pratiques professionnelles sont forcément bousculées par ce type de démarche de coconstructions de savoirs. En effet, dans ce projet, en rendant les élèves réellement acteurs, les enseignants se sont interrogés sur la relation pédagogique instaurée : «le problème aussi c'est où ils vont aller? Sans être dirigiste, là on sait pas du tout où on va ». Un autre enseignant nous avait précisé le caractère nouveau de cette démarche : "ce qui est nouveau quand même, c'est que ce sont les élèves qui choisissent le thème " (Fortin-Debart et Girault, 2007). Ainsi, c'est par la recherche-action-formation, par l'engagement de personnes volontaires motivées, par un ancrage local fort dans un contexte favorable que de tels projets peuvent se construire, dans les interstices des contraintes institutionnelles qui ne favorisent pourtant pas toujours ce type de démarche.

\section{BIBLIOGRAPHIE}

Arnstein, S.R. (1969). A Ladder of Citizen Participation. Journal of the American Institute of Planners, 35(4), 216-224.

Audigier, F. (1999). L'éducation à la citoyenneté. Paris : INRP (Institut national de recherche pédagogique).

Audigier, F. (2006). Évaluer les effets de l'éducation sur la citoyenneté. In Direction générale de l'Enseignement scolaire (dir.), Actes du séminaire national «La citoyenneté par l'éducation » (p. 37-58). Paris : Ministère de l'Éducation nationale.

Bacqué, M-H. et Carrel, M. (2005). L'intraduisible notion d'empowerment vue au fil des politiques urbaines américaines. Territoires, 460, 32-35.

Baillon, R. (1993). Le lycée, une cité à construire. Paris : Hachette.

Barthe, Y. et Rémy, É. (1997). Comment organiser la « concertation » avec les citoyens dans les controverses techniques publiques? Deux études de cas : le débat sur l'implantation de lignes à haute tension et la gestion de déchets radioactifs à vie longue. In Giordan, A., Martinand, J.-L. et Raichvarg, D. (dir.), Actes des XIXièmes Journées Internationales sur la communication, l'éducation et la culture scientifiques et industrielles : sciences, technologies et citoyenneté (p. 75-82). Paris : Association DIRES.

Bertrand, Y. (1998). Théories contemporaines de l'éducation. Montréal : Éditions Nouvelles. Blondiaux, L. et Sintomer, Y. (2002). L'impératif délibératif. Politix, 15(57), 17-35.

Blume, S.S. (1997). The rhetoric and counter-rhetoric of a « bionic » technology. Science, Technology and Human Values, 22, 31-56.

Callon, M., Hermitte, M-A., Jacquemot, F., Rousseau, D. et Testart, J. (2007). Les citoyens au pouvoir ! Libération, lundi 26 novembre 2007. Consulté en ligne en mars 2009 sur : http:// jacques.testart.free.fr/index.php?post/texte775. 
Colin, M. (2007). Démocratie technique : que peuvent les citoyens « désintéressés »? Lettre trimestrielle VivAgoVeille, 4. Consulté en ligne en mars 2009 sur : http://www.vivagora.org/ vivagoveille/vivagoveille4.html.

Crémieux, C. (1998). La citoyenneté à l'école. Paris : Syros.

De Carlo, L. (1996). Gestion de la ville et démocratie locale. Paris : L'Harmattan.

Désautels, J. (1998). Une éducation aux technosciences pour l'action sociale. In Journées internationales de didactique des sciences de Marrakech (dir.), La recherche en didactique au service de l'enseignement (p. 9-27). Marrakech : Université Cadi Ayyad, Faculté des sciences Semlalia.

Donzelot, J. et Epstein, R. (2006). Démocratie et participation : l'exemple de la rénovation urbaine. Esprit, 326, 5-34.

Driver, R., Newton, P. et Osborne, J. (2000). Establishing the norms of scientific argumentation in classrooms. Science Education, 84, 287-312.

Fortin-Debart, C. (2006). Analyse des actions d'EEDD (éducation à l'environnement pour un développement durable) mises en œuvre dans l'enseignement scolaire. Communication présentée au Colloque international « Le développement durable sous le regard des sciences et de l'histoire : de la réflexion aux pratiques éducatives et de formation », 12 et 13 octobre 2006, Arras.

Fortin-Debart, C. et Girault, Y. (2007). Appropriation d'une problématique socio-environnementale dans le cadre d'une approche coopérative - Réflexions sur la professionnalité enseignante. Communication présentée au Colloque «Éducation à l'environnement pour un développement durable Informer, former ou éduquer? ", 7 et 8 juin 2007, IUFM de Montpellier.

Freire, P. (1972). Pedagogy of the oppressed. New York : Herder and Herder.

Girault, Y., Auzou, E. et Fortin-Debart, C. (2008). De la lecture critique du territoire à la notion d' empowerment : étude de cas d'un projet d'expertise citoyenne menée avec des adolescents en banlieue parisienne. Éducation relative à l'environnement : Regards - Recherches - Réflexions, 7, 175-197.

Habermas, J. (1962). L'espace public. Paris : Éditions Payot.

Habermas, J. (1992). L'espace public, trente après. Quaderni, 18, 161-191. Hansotte, M. (2005). Les intelligences citoyennes. Bruxelles : De Boeck Université.

Hart, P. (1992). Children's participation. From tokenism to citizenship. Florence : UNICEF (United Nations Children's Fund) Innocenti Research Centre.

Irwin, A. (2001). Constructing the scientific citizen : science and democracy in the biosciences. Public Understanding of Science, 10, 1-18.

Joliveau, T. et Amzert, M. (2001). Les territoires de la participation : problème local, question universelle? Géocarrefour, 76(3), 171-174.

Jolivet, P. (2001). Le recyclage des déchets ménagers : une figure de citoyenneté. Le Courrier de l'environnement, 44, 29-40.

Jouve, B. (2006). L'empowerment : à quelles conditions ? Pour quels objectifs ? Pouvoirs locaux, 71, 113-118.

Kolsto, S.D. (2001). Scientific literacy for citizenship : tools for dealing with the science dimension of controversial socioscientific issues. Science Education, 85, 291-310.

Legardez, A. et Simonneaux, L. (2006). L'école à l'épreuve de l'actualité. Enseigner des questions vives. Paris : ESF. 
Manin, B. (1996). Principes du gouvernement représentatif. Paris : Flammarion. Mothé, D. (2006). La grande démocratie et la petite démocratie. Esprit, 326, 34-53.

Nez, H. (2006). Démocratie participative et inclusion socio-politique : les expériences de Bobigny (France) et Barreiro (Belo Horizonte, Brésil). Gatineau : Cahier de la Chaire de recherche du Canada en développement des collectivités, série Pratiques économiques et sociales no. 30.

Paoletti, M. (2003). Nouvelles pratiques démocratiques et système politique local. In ADELS (dir.), Conseils de quartier, Mode d'emploi (p. 153-161). Paris : ADELS (Association pour la démocratie et l'éducation locale et sociale.

Perrenoud, P. (1998). Le débat et la raison. Cahiers pédagogiques, supplément n 4, 4-7.

Rawls, J. (1995). Libéralisme politique. Paris : Presses Universitaires de France.

Rawls, J. (2003). La justice comme équité. Paris : La Découverte.

Rayssac, G-L. et de La Gueronniere, C. (2006). Guide de la concertation locale. Voiron : Territorial Editions.

Robottom, I. et Hart, P. (1993). Research in Environmental Education. Engaging the debate. Victoria : Deakin University.

Roqueplo, P. (1997). Entre savoir et décision, l'expertise scientifique. Paris : Éditions INRA (Institut national de la recherche agronomique).

Sauvé, L. (1997). Pour une éducation relative à l'environnement. Montréal : Éditions Guérin.

Schnapper, D. et Bachelier, C. (2000). Qu'est-ce que la citoyenneté ? Paris : Éditions Gallimard.

Sintomer, Y. (2003). Enjeux et attentes d'une démocratie participative. In ADELS (dir.), Conseils de quartier, Mode d'emploi ( $\mathrm{p}, 137-141)$. Paris : ADELS (Association pour la démocratie et l'éducation locale et sociale.

Sintomer, Y. (2006). La démocratie participative, dynamiques et défis. La revue parlementaire, décembre 2006, 30-31.

Villemagne, C. (2005). L'éducation relative à l'environnement en milieu communautaire urbain. Un modèle théorique en émergence enrichi de l'exploration collaborative de pratiques éducatives. Thèse de doctorat inédite, Université du Québec à Montréal.

Wynne, B. (1996). Misunderstood misunderstandings : Social identities and public uptake of science. In Irwin, A. et Wynne, B. (dir.), Misunderstanding science? The public reconstruction of science and technology (p. 19-46). Londres : Cambridge University Press.

\section{RÉSUMÉS}

Dans le champ de pratique et de recherche d'une éducation relative à l'environnement (ERE), nous nous interrogeons sur les modalités d'une éducation au pouvoir, c'est-à-dire d'une éducation qui vise à donner des compétences pour participer à la vie de la cité. Pour cela, nous avons souhaité faire en premier lieu le constat des pratiques de participation citoyenne mises en œuvre à l'heure actuelle en France. Sur la base de ce constat, nous avons ensuite exploré le champ des possibles pour une ERE qui se donne comme objectif principal de permettre une réelle participation citoyenne à l'encontre de l'approche comportementaliste très dominante en France. Pour conclure, et en s'appuyant sur des expériences concrètes, nous avons tenté de mettre en avant les obstacles rencontrés mais également les leviers. 
In the field of practice and research for an environmental education, we question the modalities of an education to power, that is an education which aims to give skills for participating in the life of the City. For that purpose, we report first of all on the citizen participation practices in use now in France. On the basis of this report, we then investigate the possibilities for an environmental education whose main objective is to allow true citizen participation contrary to the predominant behaviouristic approach in France. In conclusion, and by drawing upon concrete experiences, we highlight the main obstacles identified as well as potential anchorage points.

\section{AUTEURS}

\section{CÉCILE FORTIN-DEBART}

Poursuivant ses travaux de recherche en éducation relative à l'environnement après avoir soutenu une thèse en 2003 sur le partenariat école-musée en éducation relative à

l'environnement, elle est membre de l'UMR (Unité mixte de recherche) 208 du Muséum national d'Histoire naturelle de Paris (MNHN) / IRD (Institut de recherche pour le développement) Patrimoines locaux et de l'équipe de recherche Muséologie et médiation des sciences du MNHN.

\section{YVES GIRAULT}

Professeur de didactique des sciences, il dirige l'équipe de recherche Muséologie et médiation des sciences du Muséum national d'Histoire naturelle de Paris (MNHN). Il est membre de l'UMR (Unité mixte de recherche) 208 MNHN / IRD (Institut de recherche pour le développement) Patrimoines locaux. Il coordonne actuellement plusieurs programmes de recherche en éducation relative à l'environnement. 